\title{
Strip Blowing from a Wedge at Hypersonic Speeds
}

\author{
A. F. Messiter* \\ University of Michigan, Ann Arbor, Michigan 48109 \\ and \\ M. D. Matarrese $e^{\dagger}$ \\ Scaled Composites, Inc., Mojave, California 93501
}

\begin{abstract}
Surface pressure distributions are derived when gas is injected through a strip at the surface of a thin wedge in uniform flow at high Mach number. The blowing velocities are such that the flow separates ahead of the blowing region, but the layer of blown gas remains thin. Asymptotic descriptions of the separation region and the blowing region are reviewed and extended, for weak laminar viscous interaction and a cooled surface. An example with blowing through two strips is also given.
\end{abstract}

\section{Introduction}

$\mathbf{S}$ URFACE pressure distributions on a body in high-speed flow can be drastically altered if gas is injected at the body surface, through the distortion of the flowfield as well as through the momentum flux at the surface. One case of blowing from a strip on a flat plate was studied by Smith and Stewartson. ${ }^{1}$ In this case the supersonic laminar boundary layer separates somewhat ahead of the blowing region and moves away from the surface as a free shear layer at nearly constant pressure. Between the separation region and the location where blowing begins, a small part of the injected gas moves upstream at a low velocity to supply the mass needed for entrainment in the lower part of the shear layer. ${ }^{2}$ In the neighborhood of blowing a favorable pressure gradient turns the blown gas toward the downstream direction. The layer of blown gas is still thin but gradients are small enough that viscous stresses are small, and the flow here is described by inviscid boundary-layer equations. ${ }^{3}$ Downstream of the blowing region the pressure is assumed constant and equal to its undisturbed value, with no reversed flow near the wall. ${ }^{2}$

The flow properties in the laminar free interaction at separation are nearly independent of downstream conditions, and are described asymptotically at large Reynolds numbers by a variation of the tripledeck theory. The formulation relevant to self-induced separation at supersonic speeds was given by Stewartson and Williams ${ }^{4}$ and by Neiland. ${ }^{5}$ In effect the length scales are reduced near separation, and locally the flow is approximately a rotational inviscid flow with a new thinner boundary layer close to the wall. The asymptotic form of the solution somewhat downstream of separation, as the appropriate scaled variable becomes large, was described by Stewartson and Williams. ${ }^{6}$

As the Mach number increases, the length of the local-interaction region grows, as does the thickness of the viscous sublayer, until the interaction is no longer local, when the hypersonic viscousinteraction parameter is no longer small. In different terms, if the Mach number is large, the boundary layer has only a small effect on the external flow at points sufficiently far downstream: the interaction is weak. But closer to the leading edge the streamline slopes are no longer small in comparison with the slopes of characteristics in the external flow, and the boundary-layer thickness can not be neglected in a first approximation: the interaction is strong.

If separation occurs in the weak-interaction region, the asymptotic description is still local, as explained by Brown et al. ${ }^{7}$ Moreover, the

Presented as Paper 91-0032 at the AIAA 29th Aerospace Sciences Meeting, Reno, NV, Jan. 7-10, 1991; received July 9, 1993; revision received May 25, 1994; accepted for publication May 31, 1994. Copyright (C) 1994 by the American Institute of Aeronautics and Astronautics, Inc. All rights reserved.

${ }^{*}$ Professor, Department of Aerospace Engineering. Associate Fellow AIAA

${ }^{\dagger}$ Staff Aerodynamicist. Member AIAA. scale of the interaction shrinks as the wall temperature decreases. This was noted first by Neiland ${ }^{8}$ and has been discussed further by Brown et al. ${ }^{9}$ and Kerimbekov et al. ${ }^{10}$ At high Mach numbers, if the wall is cooled, the first approximation to the boundary-layer solution satisfies a condition of zero wall temperature. This, of course, must be corrected at small distances from the wall, as explained by Neiland ${ }^{8}$ and later by Seddougui et al. ${ }^{11}$ in a discussion of the effect of wall cooling on stability. In a particular limit it is found that the triple-deck solution must be augmented, since the displacement effect of the changes in the main boundary layer is no longer of higher order than that of the sublayer. ${ }^{7-10}$ For still smaller wall temperatures, the scales continue to decrease; a detailed asymptotic description for this case has recently been given by Kerimbekov et al. ${ }^{10}$ In the blowing region, the pressure changes must be compatible in the subsonic blown gas and the supersonic outer flow. If the transverse pressure gradient remains small, the problem can be reduced to solution of an integral equation for the pressure. Results for the pressure distribution and for the location of separation were given in Ref. 1 in the case of uniform blowing.

In the present work the analysis of Smith and Stewartson ${ }^{1}$ is extended to flow past a wedge at high Mach number. A description of separation from a cooled wall similar to that of Brown et al. ${ }^{9}$ is given in terms of the physical coordinate rather than the DorodnitsynHowarth variable. A complete asymptotic flow description leads to a simpler expression for a constant in the pressure-displacement relation. This part of the present derivation, given initially in the 1991 conference version of this paper, closely resembles that of Kerimbekov et al. ${ }^{10}$ The formulation for the blowing region is the same as in Ref. 1 and is applied to some specific examples, including a case with blowing through two strips. Some representative pressure distributions are shown.

\section{Formulation}

A thin wedge of length $L$ and small half-angle $\alpha \ll 1$ is placed at zero incidence in a uniform hypersonic flow at a Mach number $M_{\infty} \gg 1$. A perfect gas is assumed, with constant specific heats. Coordinates $x^{*}$ and $y^{*}$ are measured along and normal to the upper wedge surface, respectively. Gas is injected in a direction normal to the surface from a slot occupying the region $x_{0}^{*}<x^{*}<x_{1}^{*}$, where $x_{0}^{*}>0$ and $x_{1}^{*}<L$, as shown in Fig. 1. (Slopes of shock waves and characteristics in Fig. 1 have been exaggerated in an attempt at greater clarity.) The blowing velocity is such that the flow separates from the surface at a location $x^{*}=x_{s}^{*}$ upstream of the slot. The value of $x_{s}^{*}$ is unknown in advance and is determined as part of the solution, in terms of parameters including slot length and blowing velocity. It is assumed that the added mass is not so large that the separation point has moved to the wedge vertex; i.e., $0<x_{s}^{*}<x_{0}^{*}$. Separation of the laminar boundary layer occurs through a hypersonic free interaction in a small neighborhood of $x^{*}=x_{s}^{*}$, where the pressure 


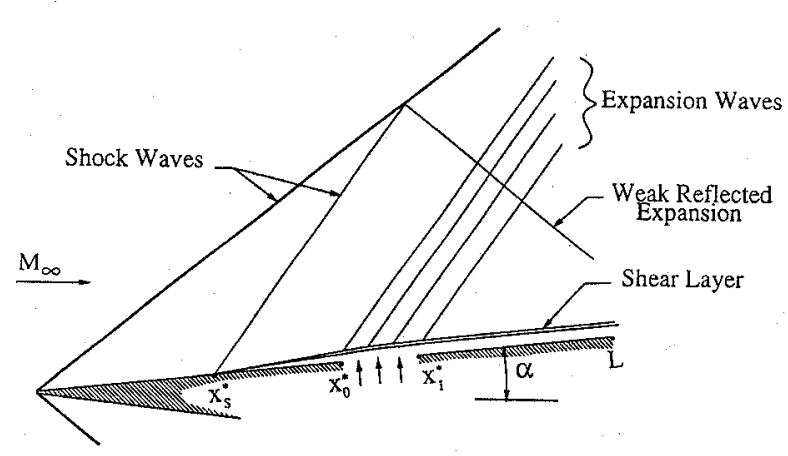

Fig. 1 Wedge flow with strip blowing.

deck analysis of the separation region. The pressure begins to drop at $x^{*}=x_{0}^{*}$, and for $x_{0}^{*}<x^{*}<x_{1}^{*}$ the blown gas is turned downstream by the favorable pressure gradient.

The velocity, pressure, density, temperature, enthalpy, and viscosity coefficient are $u, p, \rho, T, h$, and $\mu$, respectively, with corresponding undisturbed values $u_{\infty}, p_{\infty}, \rho_{\infty}, T_{\infty}, h_{\infty}$, and $\mu_{\infty}$. The Reynolds number based on $x_{s}^{*}$ and freestream quantities is $R e=$ $\rho_{\infty} u_{\infty} x_{s}^{*} / \mu_{\infty}$. The viscosity is assumed to vary as a power of the temperature $\mu / \mu_{\infty}=\left(T / T_{\infty}\right)^{\omega}$; at high temperatures the Sutherland law is recovered for $\omega=\frac{1}{2}$, whereas comparisons with certain existing results can be made if $\omega=1$.

The problem formulation is given in terms of four small dimensionless parameters: the wedge half-angle $\alpha$, the reciprocal $1 / M_{\infty}$ of the freestream Mach number, the reciprocal $R e^{-1}$ of the Reynolds number, and a nondimensional surface temperature $\theta=T_{w} /\left(M_{\infty}^{2} T_{\infty}\right)$. A limiting case is considered where $M_{\infty} \rightarrow \infty$, $\alpha \rightarrow 0, \operatorname{Re} \rightarrow \infty$, and $\theta \rightarrow 0$, such that $M_{\infty} \alpha$ is fixed (hypersonic small-disturbance theory) and $M_{\infty}^{2+\omega} R e^{-\frac{1}{2}} \rightarrow 0$ (weak viscous interaction); the order of magnitude of $\theta$ is chosen later.

The undisturbed wedge flow has shock-wave angle $\beta \sim \alpha \beta_{0}$, nondimensional pressure $p / p_{\infty} \sim M_{\infty}^{2} \alpha^{2} p_{0}$, and Mach number $M \sim \alpha^{-1} M_{0}$ found in the hypersonic small-disturbance limit from

$$
\begin{gathered}
\beta_{0}=\frac{\gamma+1}{4}+\left\{\left(\frac{\gamma+1}{4}\right)^{2}+\frac{1}{M_{\infty}^{2} \alpha^{2}}\right\}^{\frac{1}{2}} \\
p_{0}=\gamma \beta_{0}+\frac{1}{M_{\infty}^{2} \alpha^{2}} \\
\frac{1}{M_{0}^{2}}=\frac{\beta_{0}-1}{\beta_{0}} p_{0}
\end{gathered}
$$

where $\gamma$ is the ratio of specific heats and $\beta_{0} /\left(\beta_{0}-1\right)$ is the density ratio across the shock wave. Near separation these quantities characterize the uniform undisturbed wedge flow external to the boundary layer; similarly, in the blowing region the undisturbed flow above the separation streamline is the uniform wedge flow. Whereas an outgoing wave will be reflected at the shock wave, the strength of the reflected wave is numerically small in comparison with that of the incident wave; although these reflections are not weak in an asymptotic sense for the limiting case considered here, they will nevertheless be neglected. For $x_{s}^{*}$ close enough to $x_{0}^{*}$, the reflected wave reaches the separated shear layer at a location downstream of the slot, as depicted in Fig. 1, and causes a small outward bending of the shear layer at that location. For smaller $\dot{x}_{s}^{*}$, this bending can occur above the slot; the turning angle can be calculated from the strength of the incoming wave.

\section{Separation Region}

The boundary-layer equations, plus the equation of state and the viscosity law, can be written as

$$
\begin{gathered}
\frac{\partial(\rho u)}{\partial x^{*}}+\frac{\partial(\rho v)}{\partial y^{*}}=0 \\
\rho\left(u \frac{\partial u}{\partial x^{*}}+v \frac{\partial u}{\partial y^{*}}\right)+\frac{\mathrm{d} p}{\mathrm{~d} x^{*}}=\frac{\partial}{\partial y^{*}}\left(\mu \frac{\partial u}{\partial y^{*}}\right)+\cdots
\end{gathered}
$$

$$
\begin{gathered}
\rho\left(u \frac{\partial h}{\partial x^{*}}+v \frac{\partial h}{\partial y^{*}}\right)-u \frac{\mathrm{d} p}{\mathrm{~d} x^{*}}=\frac{\partial}{\partial y^{*}}\left(\frac{\mu}{\operatorname{Pr}} \frac{\partial h}{\partial y^{*}}\right)+\mu\left(\frac{\partial u}{\partial y^{*}}\right)^{2}+\cdots \\
p=\rho R T, \quad \mu / \mu_{\infty}=\left(T / T_{\infty}\right)^{\omega}
\end{gathered}
$$

where $\operatorname{Pr}$ is the Prandtl number and $R$ is the gas constant. At the wedge surface $y^{*}=0$ it is required that

$$
u=v=0, \quad . \quad T=T_{w}=\text { const }
$$

For large $M_{\infty}$, the boundary layer has thickness equal to the displacement thickness $\delta^{*}$, since the mass flow in the high-temperature boundary layer is small. ${ }^{12}$ The interaction parameter $\chi$ measures the ratio of a typical streamline slope in the boundary layer to the slope of a characteristic in the inviscid wedge flow. Here $\chi$ is defined for a length $x_{s}^{*}$ by

$$
\chi=\frac{M_{0}}{\alpha} \delta, \quad \delta=\frac{M_{\infty}^{\omega}}{\alpha p_{0}^{\frac{1}{2}} \operatorname{Re}^{\frac{1}{2}}}
$$

The displacement thickness is $\delta^{*}=\left(\right.$ const) $x_{s}^{*} \delta$; if $\omega=1$, the constant factor is $0.332(\gamma-1)$. The product $M_{\infty}^{2} \alpha^{2}$ is considered fixed in the limit, but it is possible to recover the results for a flat plate when $M_{\infty} \alpha \rightarrow 0$, since in that case $M_{\infty}^{2} \alpha^{2} p_{0} \rightarrow 1$ and $M_{0} / \alpha \rightarrow M_{\infty}$. Thus for a flat plate, $\chi$ as defined here reduces to the usual expression $M_{\infty}^{2+\omega} / R e^{\frac{1}{2}}$. If $\chi$ is small, the boundary-layer thickness is small in comparison with the wedge thickness except at points very close to the leading edge, and the interaction between the boundary layer and the external inviscid flow is weak, except near the vertex. In the following, it will be assumed that the interaction is weak; that is, $\chi \rightarrow 0$. The size of $\theta$ in terms of $\chi$ will be chosen later.

The undisturbed boundary-layer profiles are given by

$$
u / u_{\infty}=U_{0}(Y)+\cdots, \quad T / T_{\infty}=M_{\infty}^{2} T_{0}(Y)+\cdots
$$

where $Y=y^{*} /\left(x_{s}^{*} \delta\right)$ is the boundary-layer coordinate. Since the boundary-layer thickness may be set equal to the displacement thickness, the edge of the boundary layer is located at $Y=\delta^{*} /\left(x_{s}^{*} \delta\right)$. In the inviscid flow outside the boundary layer, $u / u_{\infty} \sim 1$ and $T / T_{\infty}=\mathcal{O}(1)$. Thus $U_{0} \rightarrow 1$ and $T_{0} \rightarrow 0$ as $Y \rightarrow \delta^{*} /\left(x_{s}^{*} \delta\right)$; whereas, from Eq. (8), $U_{0} \rightarrow 0$ and $T_{0} \rightarrow 0$ as $Y \rightarrow 0$; if $P r=1$, for example, then $T_{0}=(\gamma-1) U_{0}\left(1-U_{0}\right) / 2$. As $Y \rightarrow 0$, it follows from Eqs. (5) and (6) that $T_{0}^{\omega} U_{0}^{\prime} \sim \lambda=$ const and $T_{0}^{\omega} T_{0}^{\prime} \sim \lambda_{T}=$ const, so that $T_{0} \sim\left[(\omega+1) \lambda_{T} Y\right]^{1 /(\omega+1)}$ and $U_{0} \sim\left(\lambda / \lambda_{T}\right) T_{0}$ as $Y \rightarrow 0$. On the other hand, at the wall $T=T_{w} \ll M_{\infty}^{2} T_{\infty}$, and the profiles must be modified when $Y=\mathcal{O}\left(\theta^{\omega+1}\right)$ to have the form

$$
u / u_{\infty}=\theta \bar{U}_{0}(\bar{Y})+\cdots, \quad T / T_{\infty}=\theta M_{\infty}^{2} \bar{T}_{0}(\bar{Y})+\cdots
$$

where $\vec{Y}=Y / \theta^{\omega+1}$ and

$$
\bar{T}_{0}^{\omega} \bar{U}_{0}^{\prime}=\lambda, \quad \bar{T}_{0}^{\omega} \bar{T}_{0}^{\prime}=\lambda_{T}, \quad \vec{U}_{0}(0)=\bar{T}_{0}(0)-1=0
$$

so that

$$
\bar{T}_{0}=\left[1+(\omega+1) \lambda_{T} \bar{Y}\right]^{1 /(\omega+1)}, \quad \bar{U}_{0}=\left(\lambda / \lambda_{T}\right)\left(\bar{T}_{0}-1\right)
$$

This formulation appears to have been given first by Neiland ${ }^{8}$; these modifications near the surface have also been noted by Seddougui et al., ${ }^{11}$ Brown et al., ${ }^{9}$ and Kerimbekov et al. ${ }^{10}$

In the neighborhood of separation the reference length in the flow direction is small, and the proper streamwise coordinate is

$$
x=\frac{1}{\Delta}\left(\frac{x^{*}}{x_{s}^{*}}-1\right)
$$

where $\Delta=\Delta(\chi, \theta) \ll 1$ and is to be determined. In most of the boundary layer, for the case to be considered here, the perturbations in $u$ and $p$ are chosen to be of the same order of magnitude, say $\mathcal{O}(\varepsilon)$, where $\varepsilon=\varepsilon(\chi, \theta)$. A consequence of this choice will be a relation between the orders of magnitude of $\theta$ and $\chi$. The flow variables are then expanded for $x=\mathcal{O}(1)$ and $Y=\mathcal{O}(1)$ in the 
form

$$
\begin{gathered}
\frac{u}{u_{\infty}}=U_{0}(Y)+\varepsilon U_{1}(x, Y)+\cdots \\
\frac{v}{u_{\infty}}=\frac{\delta}{\Delta} \varepsilon V_{1}(x, Y)+\cdots \\
p /\left(M_{\infty}^{2} \alpha^{2} p_{0} p_{\infty}\right)=1+\varepsilon \gamma P_{1}(x, Y)+\cdots \\
\frac{T}{M_{\infty}^{2} T_{\infty}}=T_{0}(Y)+\varepsilon T_{1}(x, Y)+\cdots \\
\frac{\rho}{\alpha^{2} p_{0} \rho_{\infty}}=\rho_{0}(Y)+\varepsilon \rho_{1}(x, Y)+\cdots
\end{gathered}
$$

The perturbation quantities satisfy linear differential equations

$$
\begin{gathered}
\rho_{0}\left(U_{1 x}+V_{1 Y}\right)+U_{0} \rho_{1 x}+\rho_{0}^{\prime} V_{1}=0 \\
U_{0} U_{1 x}+U_{0}^{\prime} V_{1}+P_{1 x} / \rho_{0}=0, \quad P_{1 Y}=0 \\
U_{0} T_{1 x}+T_{0}^{\prime} V_{1}-(\gamma-1) U_{0} P_{1 x} / \rho_{0}=0
\end{gathered}
$$

and the equation of state gives

$$
\rho_{0} T_{0}=1, \quad \gamma P_{1}=\frac{\rho_{1}}{\rho_{0}}+\frac{T_{1}}{T_{0}}
$$

Thus the viscous forces are of higher order than the convection and pressure terms, and the equations for the first approximation describe small disturbances to an inviscid rotational flow.

The solutions to Eqs. (18-21) are

$$
\begin{gathered}
\frac{V_{1}}{U_{0}}=P_{1}^{\prime} \int_{0}^{Y}\left(\frac{T_{0}}{U_{0}^{2}}-1\right) \mathrm{d} Y-A_{1}^{\prime} \\
U_{1}=-P_{1} U_{0}^{\prime} \int_{0}^{Y}\left(\frac{T_{0}}{U_{0}^{2}}-1\right) \mathrm{d} Y-P_{1} \frac{T_{0}}{U_{0}}+U_{0}^{\prime} A_{1} \\
T_{1}=-P_{1} T_{0}^{\prime} \int_{0}^{Y}\left(\frac{T_{0}}{U_{0}^{2}}-1\right) \mathrm{d} Y+(\gamma-1) T_{0} P_{1}+T_{0}^{\prime} A_{1}
\end{gathered}
$$

where $P_{1}=P_{1}(x)$ and $A_{1}=A_{1}(x) ; U_{0} / T_{0}^{\frac{1}{2}}$ is equal to the local Mach number. The integral exists, since $U_{0}$ and $T_{0}$ are $\mathcal{O}\left(Y^{1 /(\omega+1)}\right)$ as $Y \rightarrow 0$; thus as $Y \rightarrow 0$,

$$
\begin{gathered}
V_{1} \sim-U_{0}(Y) A_{1}^{\prime}(x), \quad U_{1} \sim U_{0}^{\prime}(Y) A_{1}(x) \\
T_{1} \sim T_{0}^{\prime}(Y) A_{1}(x)
\end{gathered}
$$

When $Y=\mathcal{O}\left(\theta^{\omega+1}\right)$, the proper coordinate is $\bar{Y}$ rather than $Y$, and the profiles $U_{0}(Y)$ and $T_{0}(Y)$ must be replaced by $\theta \bar{U}_{0}(\bar{Y})$ and $\theta \bar{T}_{0}(\bar{Y})$. The convection terms are now the largest terms in Eqs. (19) and (20), so that the solutions for $\bar{Y}=\mathcal{O}(1)$ have the same form as in Eq. (25) but in terms of the barred profiles. Then as $\bar{Y} \rightarrow 0$

$$
\begin{gathered}
\frac{v}{u_{\infty}} \sim-\frac{\delta}{\Delta} \varepsilon \theta^{-\omega} \lambda Y A_{1}^{\prime}, \quad \frac{u}{u_{\infty}} \sim \theta^{-\omega} \lambda\left(Y+\varepsilon A_{1}\right) \\
\frac{T-T_{w}}{M_{\infty}^{2} T_{\infty}} \sim \theta^{-\omega} \lambda_{T}\left(Y+\varepsilon A_{1}\right)
\end{gathered}
$$

As in the conventional triple-deck theory, viscous forces can no longer be neglected near the surface, and different asymptotic representations are required in a thin sublayer where $Y$ and $\vec{Y}$ are small. An inner variable $y$ is defined by

$$
y=\frac{Y}{\zeta}=\frac{1}{\zeta} \frac{y^{*}}{x_{s}^{*} \delta}
$$

where $\zeta=\zeta(\chi, \theta) \ll 1$, and it will be found that $\zeta$ also satisfies the stronger condition $\zeta \ll \theta^{\omega+1}$; thus, the sublayer is thin enough that the solutions there should match with the solutions given by Eq. (26). The limit process for the sublayer is chosen such that all terms in the boundary-layer momentum equation are of the same order, the largest terms in the solutions for $u$ are matched, and the solutions for $p$ are matched. Since the pressure perturbation is $\mathcal{O}(\varepsilon)$ in the sublayer as well as in the main boundary layer, these conditions lead to expansions in the form

$$
\begin{gathered}
\frac{u}{u_{\infty}}=\varepsilon^{\frac{1}{2}} \theta^{\frac{1}{2}} u_{1}(x, y)+\cdots \\
\frac{v}{u_{\infty}}=\lambda \delta \varepsilon^{-\frac{1}{2}} \theta^{\frac{1}{2}} v_{1}(x, y)+\cdots \\
\frac{p}{M_{\infty}^{2} \alpha^{2} p_{0} p_{\infty}}=1+\varepsilon \gamma p_{1}(x, y)+\cdots \\
\frac{T}{M_{\infty}^{2} T_{\infty}}=\theta+\cdots
\end{gathered}
$$

where the scales $\Delta$ and $\zeta$ are found in terms of $\varepsilon$ as

$$
\Delta=\lambda^{-2} \varepsilon^{\frac{3}{2}} \theta^{\omega+\frac{1}{2}}, \quad \zeta=\lambda^{-1} \varepsilon^{\frac{1}{2}} \theta^{\omega+\frac{1}{2}}
$$

The perturbation quantities satisfy the incompressible boundarylayer equations

$$
\begin{gathered}
u_{1 x}+v_{1 y}=0 \\
u_{1} u_{1 x}+v_{1} u_{1 y}+p_{1 x}=u_{1 y y}, \quad p_{1 y}=0
\end{gathered}
$$

with boundary conditions $u_{1}=v_{1}=0$ at $y=0$ and initial condition $u_{1} \sim y$ as $x \rightarrow-\infty$. As $y \rightarrow \infty$,

$$
u_{1} \sim y+A, \quad v_{1} \sim-A^{\prime} y
$$

where the function $-A(x)$ implies an effective shift of the origin for $y$, and so represents a scaled change in displacement thickness of the sublayer. The form of the expansions defined by Eqs. (28-32) is independent of the relative sizes of the small parameters $\chi$ and $\theta$.

The interaction of the boundary layer with the external flow determines a relation between $p_{1}$ and $A_{1}$, as well as a definition for $\varepsilon$ and, therefore, expressions for $\Delta$ and $\zeta$. In the flow outside the boundary layer, the pressure and the velocity components satisfy a linear wave equation, for $x^{*}-x_{s}^{*}=O\left(x_{s}^{*} \Delta\right)$ and $y^{*}=\mathcal{O}\left(x_{s}^{*} \alpha \Delta / M_{0}\right)$. If only outgoing waves are present locally, the result at the edge of the boundary layer is the usual linear-theory relation between the pressure perturbation and the streamline slope. Since these quantities are continuous at the edge of the boundary layer, the solutions in the main boundary layer evaluated at $Y=\delta^{*} /\left(x_{s}^{*} \delta\right)$ must satisfy this condition. It follows that

$$
\varepsilon P_{1}+\cdots=\chi(\varepsilon / \Delta) V_{1}+\cdots
$$

at $Y=\delta^{*} /\left(x_{s}^{*} \delta\right)$, and so $\Delta=\mathcal{O}(\chi)$. Matching the second terms in $u$ from Eqs. (26) and (35) gives $\varepsilon=\mathcal{O}\left(\theta^{2 \omega+1}\right)$ and $A_{1}=$ (const) $A$. Combining with Eq. (32) then shows that $\theta=\mathcal{O}\left(\chi^{1 /(4 \omega+2)}\right)$ for the case considered. It is convenient to introduce constant factors in such a way that the pressure-displacement relation found from Eqs. (36) and (22) contains a single parameter $Q$. Since also $p_{1}=P_{1}$, the results are

$$
\begin{gathered}
p_{1}=-A^{\prime}-\frac{1}{Q} p_{1}^{\prime} \\
\varepsilon=\lambda^{\frac{1}{2}} \chi^{\frac{1}{2}}, \quad A_{1}(x)=\theta^{\omega+\frac{1}{2}} \lambda^{-\frac{5}{4}} \chi^{-\frac{1}{4}} A(x)
\end{gathered}
$$

where the parameter $Q$ is defined by

$$
Q I=\theta^{\omega+\frac{1}{2}} \lambda^{-\frac{5}{4}} \chi^{-\frac{1}{4}}, \quad I=\int_{0}^{\infty} T_{0}\left(1-\frac{T_{0}}{U_{0}^{2}}\right) \mathrm{d} \eta
$$

The integral $I$ in Eq. (39) has been rewritten in terms of a Dorodnitsyn-Howarth variable $\eta$ defined by $\mathrm{d} Y=T_{0} \mathrm{~d} \eta$; as $\eta \rightarrow 0$, both $T_{0}$ and $U_{0}$ are $\mathcal{O}\left(\eta^{-1 / \omega}\right)$. The streamwise length scale $\Delta=$ $\mathcal{O}(\chi)$ has been made specific by the choice $\Delta=Q I \chi$, for convenience in recovering the case of large $Q$, as noted subsequently; it follows also that $\zeta=Q I \varepsilon$. Equation (37) serves as an additional boundary condition for Eqs. (33) and (34) as $y \rightarrow \infty$, and can be regarded as an interaction condition. 


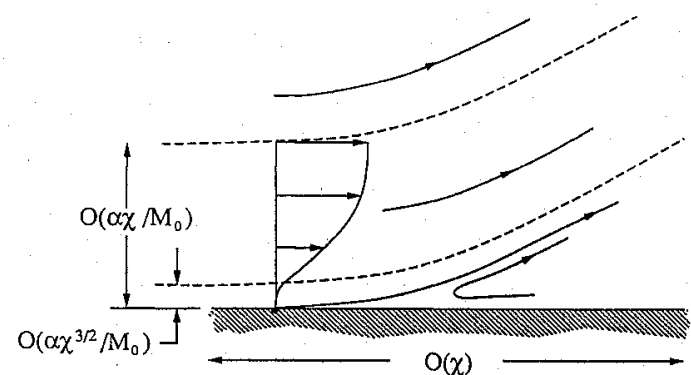

Fig. 2 Flow structure near separation.

Thus, the special case in which the displacement effects of the viscous sublayer and the main part of the boundary layer are of the same order of magnitude corresponds to a limit such that $\chi \rightarrow 0$ and $\theta \rightarrow 0$ with $\theta^{\omega+\frac{1}{2}} / \chi^{\frac{1}{4}}$ held fixed. In this limit, the scalings in Eqs. (14), (27), and (32) are, as already noted,

$$
\Delta=Q I \chi, \quad \zeta=Q I \varepsilon
$$

and so the coordinates become

$$
\left(x^{*}-x_{s}^{*}\right) / x_{s}^{*}=Q I \chi x, \quad Y=Q I \varepsilon y
$$

The flow regions are sketched in Fig. 2. The length scales for the local external flow are the same as for the boundary layer: for $x^{*}-x_{s}^{*}=\mathcal{O}\left(x_{s}^{*} \Delta\right)$, disturbances in the outer flow extend only to a distance $y^{*}=\mathcal{O}\left(\delta^{*}\right)$ and not to a still larger distance as in the usual triple-deck theory. Since $\zeta=\mathcal{O}\left(\chi^{\frac{1}{2}}\right)$ and $\chi=\mathcal{O}\left(\theta^{4 \omega+2}\right)$, it is seen that $\zeta \ll \theta^{\omega+1}$, as anticipated following Eq. (27). Thus, the interaction occurs in a streamwise length $\mathcal{O}\left(\chi x_{s}^{*}\right)$, and the sublayer thickness is $\mathcal{O}\left(\chi^{\frac{1}{2}} \delta^{*}\right)$; if $\omega=1$, the nondimensional wall temperature is $\theta=\mathcal{O}\left(\chi^{\frac{1}{6}}\right)$, and the region where corrections to $U_{0}$ and $T_{0}$ are required has thickness $\mathcal{O}\left(\chi^{\frac{1}{3}} \delta^{*}\right)$.

Parameters equivalent to $Q$ have also been given in Refs. 7-11; in Refs. 7 and 11 the exponent $\omega$ was taken equal to one. The interaction relation (37) has been given by Neiland, ${ }^{8}$ by Brown et al., ${ }^{7}$ by Brown et al., ${ }^{9}$ and by Kerimbekov et al. ${ }^{10}$ In the second of these, the coefficient of $p_{1}^{\prime}$ has the same dependence on $\chi, \theta$ and on the profile shapes, and is of order one because $\gamma-1 \ll 1$ rather than $\theta \ll 1$. Brown et al. ${ }^{9}$ used a Dorodnitsyn-Howarth variable, obtained a divergent integral, and gave a more complicated right-hand side.

For a constant-pressure boundary layer with $\operatorname{Pr}=1$, the integral in Eq. (39) can be evaluated by setting $T_{0}=(\gamma-1) U_{0}\left(1-U_{0}\right) / 2$ and taking numerical values from the Blasius solution

$$
I=\frac{1}{4}\left(\gamma^{2}-1\right) \cdot 0.664-\left(\frac{\gamma-1}{2}\right)^{2} \cdot 1.721
$$

If $\gamma=1.4$, the value is 0.0905 . Thus for this profile, the main boundary layer behaves as a supersonic flow, since from Eq. (22), with interaction omitted, the displacement thickness decreases as the pressure increases. On the other hand, since $-A$ is a sublayer displacement thickness, the first term $-A^{\prime}$ is positive in Eq. (37). Values of plateau pressure $p_{1 f}$ given in Ref. 9 are $p_{1 f}=p_{1}(\infty)=$ $1.809,1.681$, and 1.564 when the value of $Q$ in Eq. (37) is $\infty, 1$, and 0.5 , respectively.

For large $Q$, the forms of the sublayer expansions in Eqs. (28$31)$ are unchanged. In the main boundary layer, the pressure perturbation remains $\mathcal{O}(\varepsilon)$, but the velocity, temperature, and density perturbations in Eqs. (15) and (17) are $\mathcal{O}(Q \varepsilon)$, because it follows from Eqs. (38) and (39) that $A_{1}=\mathcal{O}(Q)$, and the choice $\Delta=Q I \chi$ gives $V_{1}\left(x, \delta^{-1} \delta^{*} / x_{s}^{*}\right)=Q I P_{1}(x)$. As a consequence, the pressuredisplacement relation in Eq. (37) becomes $p_{1}=-A^{\prime}$. If $\omega=1$, the formulation for the first approximation is then identical to that of conventional triple-deck theory in the limit as $M_{\infty} \rightarrow \infty$.

On the other hand, Eq. (37) requires modification if $Q \rightarrow 0$, when the displacement effect due to the main boundary layer becomes large compared to that of the sublayer. The factor $\Delta$ introduced in the scaling (14) for $x^{*}$, and defined in Eq. (40), now should not include the factor $Q$, but should be simply $\Delta=\chi$, with the factor $I$ remaining in the interaction condition so that in the limit Eq. (37) is replaced by $I p_{1}^{\prime}+p_{1}=0$. A detailed description of the limiting flow as $Q \rightarrow 0$ has recently been given by Kerimbekov et al. ${ }^{10}$ for the hypersonic boundary-layer interaction at a shallow compression corner. They considered both the supercritical case $I>0$ and the subcritical case $I<0$, concluding from the sign change in the exponential solution for $p_{1}$ that the pressure rise occurs primarily downstream and upstream of the corner, respectively. In each case smaller length scales become important and a still more complicated asymptotic structure arises. For the present free-interaction problem it seems reasonable to anticipate that a sign change in the interaction relation would affect primarily the flow details in the separation region and that the pressure rise would again occur primarily downstream or upstream of the separation point, respectively, when $I>0$ or $I<0$. If this is true as $Q \rightarrow 0$, a corresponding shift in the location of the pressure rise would also be expected to persist when $Q$ is not small, with the changes occurring earlier for $I<0$ than for $I>0$. The present solutions are restricted to values of $Q$ that are not small, since the case $Q \rightarrow 0$ has not been considered further here.

\section{Blowing Region}

Gas is injected from the surface in the range $x_{0}^{*}<x^{*}<x_{1}^{*}$, with separation occurring farther upstream, at $x^{*}=x_{s}^{*}$, where $0<$ $x_{s}^{*}<x_{0}^{*}$. Downstream of separation the separated boundary layer moves away from the surface as a thin free shear layer at nearly constant pressure. A low-speed backflow, ${ }^{2}$ accelerated by a higherorder pressure gradient, provides the small amount of mass required for entrainment in the thin shear layer for $x_{s}^{*}<x^{*}<x_{0}^{*}$. Most of the added mass, however, is turned downstream by the favorable pressure gradient for $x_{0}^{*}<x^{*}<x_{1}^{*}$, as indicated in Fig. 3. Since the mass entrained is small in comparison with the total mass added at the wedge surface, the streamlines entering the shear layer originate at points very close to the beginning of blowing.

The slope of the separated shear layer is $\mathrm{d} y^{*} / \mathrm{d} x^{*}=\lambda^{\frac{1}{2}} \chi^{\frac{1}{2}}$ $p_{1 f} \alpha / M_{0}$. At $x^{*}=x_{0}^{*}$, the beginning of blowing, the distance from the surface to the shear layer is $y^{*}=\tilde{\delta}_{0}$, where

$$
\tilde{\delta}_{0}=\left(\alpha / M_{0}\right) \lambda^{\frac{1}{2}} \chi^{\frac{1}{2}} p_{1 f}\left(x_{0}^{*}-x_{s}^{*}\right)
$$

Suitable coordinates for the blowing region are, therefore,

$$
\tilde{x}=\frac{x^{*}-x_{0}^{*}}{x_{0}^{*}-x_{s}^{*}}, \quad \tilde{y}=\frac{y^{*}}{\tilde{\delta}_{0}}
$$

where $0<\tilde{x}<\tilde{x}_{1}$ and $\tilde{x}_{1}$ is the value of $\tilde{x}$ at $x^{*}=x_{1}^{*}$. The shear layer has a thickness small in comparison with $\tilde{\delta}_{0}$ and is defined by $\tilde{y}=\tilde{\Delta}(\tilde{x})$, with $\tilde{\Delta}(0)=1$. The solutions are expanded in the form

$$
\begin{gathered}
\frac{u}{u_{\infty}}=\frac{\tilde{T}_{w}^{\frac{1}{2}}}{M_{\infty}} \lambda^{\frac{1}{4}} \chi^{\frac{1}{4}} p_{1 f}^{\frac{1}{2}} \tilde{u}_{1}(\tilde{x}, \tilde{y})+\cdots \\
\frac{v}{u_{\infty}}=\frac{\tilde{T}_{w}^{\frac{1}{2}}}{M_{\infty}} \frac{\tilde{\delta}_{0}}{x_{0}^{*}-x_{s}^{*}} \lambda^{\frac{1}{4}} \chi^{\frac{1}{4}} p_{1 f}^{\frac{1}{2}} \tilde{v}_{1}(\tilde{x}, \tilde{y})+\cdots \\
\frac{p}{M_{\infty}^{2} \alpha^{2} p_{0} p_{\infty}}=1+\lambda^{\frac{1}{2}} \chi^{\frac{1}{2}} \gamma p_{1 f} \tilde{p}_{1}(\tilde{x}, \tilde{y})+\cdots \\
\frac{T}{T_{\infty}}=\tilde{T}_{w}+\cdots
\end{gathered}
$$

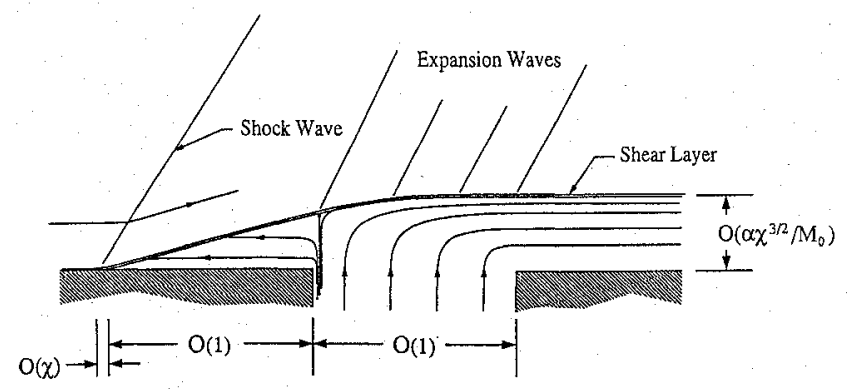

Fig. 3 Some flow details for strip blowing. 
where the temperature is nearly constant because the local Mach number is small. If the temperature of the injected gas were the same as that of the surface upstream of the slot, then $\tilde{T}_{w}$ would be equal to $M_{\infty}^{2} \theta$. The differential equations for the flow perturbations are inviscid boundary-layer equations

$$
\begin{gathered}
\frac{\partial \tilde{u}_{1}}{\partial \tilde{x}}+\frac{\partial \tilde{v}_{1}}{\partial \tilde{y}}=0 \\
\tilde{u}_{1} \frac{\partial \tilde{u}_{1}}{\partial \tilde{x}}+\tilde{v}_{1} \frac{\partial \tilde{u}_{1}}{\partial \tilde{y}}=-\frac{\partial \tilde{p}_{1}}{\partial \tilde{x}},
\end{gathered}
$$

The surface boundary condition in the blowing region is

$$
\tilde{v}_{1}=\tilde{v}_{1 w}(\tilde{x}) \quad \text { at } \quad \tilde{y}=0, \quad 0<\tilde{x}<\tilde{x}_{1}
$$

A stream function is defined by

$$
\frac{\partial \psi}{\partial \tilde{y}}=\tilde{u}_{1} \quad \frac{\partial \psi}{\partial \tilde{x}}=-\tilde{v}_{1}
$$

Integration of the first of Eqs. (52) gives

$$
\tilde{y}=\int_{\psi_{w}}^{\psi} \frac{\mathrm{d} \psi}{\tilde{u}_{1}}=-\int_{\tilde{x}}^{\tilde{x}_{w}} \frac{\tilde{v}_{1 w}}{\tilde{u}_{1}} \mathrm{~d} \tilde{x}_{w}
$$

where a streamline is denoted by the location $\tilde{x}_{w}(\psi)$ whereupon it leaves the wall in the blowing region, and so $\mathrm{d} \psi / \mathrm{d} \tilde{x}_{w}=-\tilde{v}_{1 w}$, with $\tilde{x}_{w}(0)=0$. Equation (50) then implies the Bernoulli equation

$$
\tilde{p}_{1}(\tilde{x})+\frac{1}{2} \tilde{u}_{1}^{2}\left(\tilde{x}, \tilde{x}_{w}\right)=\tilde{p}_{1}\left(\tilde{x}_{w}\right)
$$

Outgoing waves that originate in the free-interaction region will be reflected from the shock wave, but the reflections will be numerically quite weak. For simplicity these reflected waves will be ignored; their effects could be added later if it were desired. (The location and strength of the waves reflected from the shock can be calculated, and incoming waves at the free shear layer can then be taken into account. If $\left(x_{1}^{*}-x_{0}^{*}\right) / x_{0}^{*}$ is small enough, as suggested in Fig. 1, the correction will be entirely downstream of the slot.) Since the pressure above the shear layer is then linear in the slope,

$$
\tilde{\Delta}(\tilde{x})=1+\int_{0}^{\tilde{x}} \tilde{p}_{1} \mathrm{~d} \tilde{x}
$$

and $\tilde{p}_{1}(0)=1$, since the pressure at the beginning of blowing is equal to the plateau pressure achieved downstream of separation. Combining Eqs. (53) and (54), also

$$
\tilde{\Delta}(\tilde{x})=\frac{1}{2^{\frac{1}{2}}} \int_{0}^{\tilde{x}} \frac{\tilde{v}_{1 w}\left(\tilde{x}_{w}\right) \mathrm{d} \tilde{x}_{w}}{\left\{\tilde{p}_{1}\left(\tilde{x}_{w}\right)-\tilde{p}_{1}(\tilde{x})\right\}^{\frac{1}{2}}}
$$

For the special case $\tilde{v}_{1 w}=$ const, the solution ${ }^{1}$ can be found as a series in the inverse form

$$
\frac{\mathrm{d} \tilde{x}}{\mathrm{~d} \tilde{p}_{1}}=-\sum_{n=0}^{\infty} A_{n}\left(1-\tilde{p}_{1}\right)^{(n-1) / 2}
$$

where $A_{0}=2^{\frac{1}{2}} /\left(\pi \tilde{v}_{1 w}\right)$ and $A_{n}$ satisfies a recursion relation for $n>0$, namely,

$$
A_{n}=\frac{(2 / \pi)^{\frac{1}{2}}}{\tilde{v}_{1 w}} \frac{\Gamma(n / 2)}{\Gamma[(n+1) / 2]}\left(A_{n-1}-A_{n-3}\right)
$$

with $A_{k}$ taken to be zero for $k<0$. Evaluation of Eq. (55) gives

$$
=1+\sum_{n=0}^{\infty} \frac{4}{(n+1)(n+3)} A_{n}\left(1+\frac{n+1}{2} \tilde{p}_{1}\right)\left(1-\tilde{p}_{1}\right)^{(n+1) / 2}
$$

Following Smith and Stewartson, ${ }^{1}$ it is assumed that there is no separation at the end of blowing, where $\tilde{x}=\tilde{x}_{1}$. Then farther downstream, for $\tilde{x}>\tilde{x}_{1}$, the flow must be parallel to the surface, for there would otherwise appear to be a contradiction: either the low-speed flow adjacent to the surface decelerates and the pressure begins to rise again, or this flow accelerates and the separation streamline from $x^{*}=x_{s}^{*}$ has negative slope. It is, therefore, assumed that $\tilde{p}_{1}=0$ at $\tilde{x}=\tilde{x}_{1}$. Integration of Eq. (57) then gives

$$
\tilde{x}_{1}=\frac{x_{1}^{*}-x_{0}^{*}}{x_{0}^{*}-x_{s}^{*}}=2 \sum_{n=0}^{\infty} \frac{1}{n+1} A_{n}
$$

Since the $A_{n}$ depend on $\tilde{v}_{1 w}$, Eq. (60) specifies the location of separation $x^{*}=x_{s}^{*}$ in terms of the scaled blowing velocity $\tilde{v}_{1 w}$. If $\tilde{v}_{1 w}$ is small, separation occurs at a point very close to the beginning of blowing ${ }^{1}$

$$
\frac{x_{0}^{*}-x_{s}^{*}}{x_{1}^{*}-x_{0}^{*}}=\frac{3 \tilde{v}_{1 w}}{4 \sqrt{2}} \exp \left\{\frac{-2}{3 \pi \tilde{v}_{1 w}^{2}}\right\}+\cdots
$$

If, instead, $\tilde{v}_{1 w}$ is large, a first approximation for the location of separation is

$$
\frac{x_{s}^{*}}{x_{0}^{*}}=1-\frac{x_{1}^{*}-x_{0}^{*}}{x_{0}^{*}} \frac{\pi \tilde{v}_{1 w}}{2^{\frac{3}{2}}}\left[1+\mathcal{O}\left(\tilde{v}_{1 w}^{-1}\right)\right]
$$

where it is understood that the length $x_{1}^{*}-x_{0}^{*}$ of the blowing region is $\mathcal{O}\left(\tilde{v}_{1 w}^{-1} x_{0}^{*}\right)$ and is small enough that $x_{s}^{*}>0$. Thus, for large $\tilde{v}_{1 w}$ the distance from separation to the slot is proportional to the product of blowing velocity and slot width; i.e., $x_{0}^{*}-x_{s}^{*}$ is proportional to the rate at which mass is added. The corresponding pressure distribution in the blowing region $x_{0}^{*}<x^{*}<x_{1}^{*}$ is found from

$$
\tilde{p}_{1}=1-\left(\frac{\tilde{x}}{\tilde{x}_{1}}\right)^{2}\left\{1-\frac{1}{2^{\frac{3}{2}} \tilde{v}_{1 w}}\left[4 \frac{\tilde{x}}{\tilde{x}_{1}}-\left(\frac{\tilde{x}}{\tilde{x}_{1}}\right)^{3}-3\right]\right\}+\mathcal{O}\left(\tilde{v}_{1 w}^{-2}\right)
$$

Thus, the pressure is constant from separation until the beginning of blowing and to a first approximation for large $\tilde{v}_{1 w}$ is quadratic in $x^{*}-x_{0}^{*}$ in the blowing region; the length $\mathcal{O}\left(x_{s}^{*} \chi\right)$ of the separation region is small in comparison with the other lengths.

The interaction parameter $\chi$ has been defined by Eq. (9) in terms of the distance $x_{s}^{*}$ from the wedge vertex to the separation point, where $x_{s}^{*}$ depends on the location and strength of the blowing and is obtained as part of the solution. Thus, the definitions of the quantities $\tilde{\delta}_{0}, \tilde{u}_{1}, \tilde{v}_{1}$, and $\tilde{p}_{1}$ in Eqs. (43), (45), (46), and (47) also depend on the value of $x_{s}^{*}$, which is unknown in advance. For example, the initial value of $\tilde{p}_{1}$ is $\tilde{p}_{1}(0)=1$, whereas the actual pressure perturbation at the beginning of the strip is proportional to $\chi^{\frac{1}{2}} \tilde{p}_{1}(0)$, which increases as $x_{s}^{*}$ decreases. For some purposes it is preferable, instead, to think in terms of quantities that depend on a specified length, say, the distance $x_{1}^{*}$ from the wedge vertex to the end of the strip within which mass is added. This can be accomplished by replacing $\chi$ with an interaction parameter $\chi_{1}$ based on $x_{1}^{*}$ instead of $x_{s}^{*}$. Then each of the quantities $\tilde{\delta}_{0}, \tilde{u}_{1}, \tilde{v}_{1}$, and $\tilde{p}_{1}$ is multiplied by a power of $\chi / \chi_{1}=\left(x_{1}^{*} / x_{s}^{*}\right)^{\frac{1}{2}}$, as indicated in some of the figures described subsequently.

Pressure distributions found from Eq. (57) for various values of the scaled blowing velocity $\tilde{v}_{1 w}$ are shown in Fig. 4 , for $x_{0}^{*} / x_{1}^{*}=0.5$, by plots of $\left(\chi / \chi_{1}\right)^{\frac{1}{2}} \tilde{p}_{1}$ vs $x^{*} / x_{1}^{*}$; these curves are equivalent to those given in Ref. 1 . On the scale of the wedge length the pressure increase near separation appears as a jump, and the location of separation is seen to move upstream as $\tilde{v}_{1 w}$ increases. The magnitude of the pressure jump increases as separation moves forward, since the jump is proportional to $\chi^{\frac{1}{2}}$ and $\chi$ is proportional to $\left(x_{s}^{*}\right)^{-\frac{1}{2}}$. When $\tilde{v}_{1 w}=$ 0.2 , separation occurs very close to the beginning of blowing, as predicted by Eq. (61). For $\tilde{v}_{1 w}=1.0$, the pressure is close to the form for large $\tilde{v}_{1 w}$ given by Eq. (63). The scaled location of the separation streamline is plotted against $x^{*} / x_{1}^{*}$ in Fig. 5, again for several values of $\tilde{v}_{1 w}$. The slope is constant between separation and the beginning of blowing and decreases to zero at the end of the blowing region. The value of the initial slope is proportional to the pressure increase at separation and, therefore, increases as $\tilde{v}_{1 w}$ increases. 


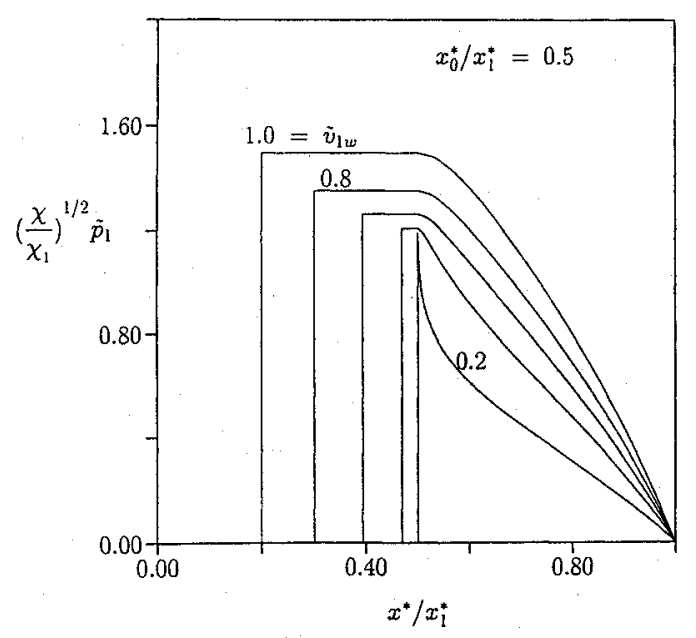

Fig. 4 Scaled pressure distribution for various scaled blowing velocities.

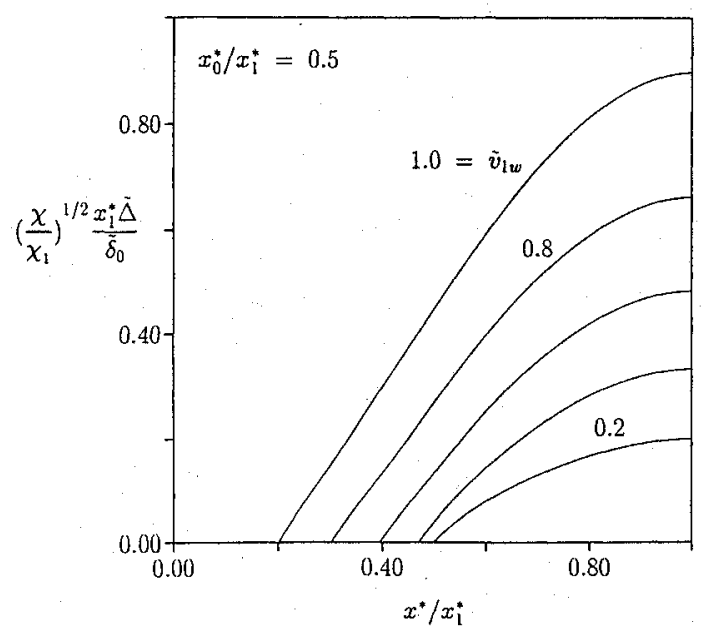

Fig. 5 Scaled location of separation streamline for various scaled blowing velocities.

The mass flow rate $\dot{m}$ per unit span is

$$
\begin{aligned}
\dot{m} & =\int_{x_{0}^{*}}^{x_{1}^{*}}[\rho v]_{\tilde{y}=0} \mathrm{~d} x^{*} \\
& =\left(M_{\infty}^{2} \alpha^{2} p_{0} \rho_{\infty}\right) \frac{\alpha}{M_{0}} \frac{u_{\infty}}{M_{\infty} \tilde{T}_{w}^{\frac{1}{2}}} \lambda^{\frac{3}{4}} \chi_{1}^{\frac{3}{4}} \gamma p_{1 f}^{\frac{3}{2}} x_{1}^{*} \dot{m}_{1}
\end{aligned}
$$

where the nondimensional mass flow $\dot{m}_{1}$ is defined by

$$
\dot{m}_{1}=\frac{\chi^{\frac{3}{4}}}{\chi_{1}^{\frac{3}{4}}} \frac{x_{1}^{*}-x_{0}^{*}}{x_{1}^{*}} \int_{0}^{1} \tilde{v}_{1 w} \mathrm{~d} \frac{\tilde{x}}{\tilde{x}_{1}}
$$

The integrated force change and the location of separation are shown in Fig. 6 in terms of $\dot{m}_{1}$. The distance between the separation point and the beginning of blowing is very small when $\dot{m}_{1}=0.2$, as is also evident in Figs. 4 and 5. This distance increases as $\dot{m}_{1}$ increases and becomes nearly linear in $\dot{m}_{1}$ when $\dot{m}_{1}$ is greater than about 0.6 , in agreement with Eq. (62). The added pressure force acting on the wedge surface has the form

$$
\Delta F=\int_{x_{0}^{*}}^{x_{1}^{*}} \Delta p \mathrm{~d} x^{*}=M_{\infty}^{2} \alpha^{2} p_{0} p_{\infty} \lambda^{\frac{1}{2}} \chi_{1}^{\frac{1}{2}} \gamma p_{1 f} x_{1}^{*} F_{1}
$$

in terms of the interaction parameter $\chi_{1}$ based on $x_{1}^{*}$; here $\Delta p$ is the difference between the surface pressure and the undisturbed wedge pressure. The scaled force change $F_{1}$ is

$$
F_{1}=\left(\frac{1}{\tilde{x}_{1}}+\int_{0}^{1} \tilde{p}_{1} d \frac{\tilde{x}}{\tilde{x}_{1}}\right) \frac{\chi^{\frac{1}{2}}}{\chi_{1}^{\frac{1}{2}}} \frac{x_{1}^{*}-x_{0}^{*}}{x_{1}^{*}}
$$

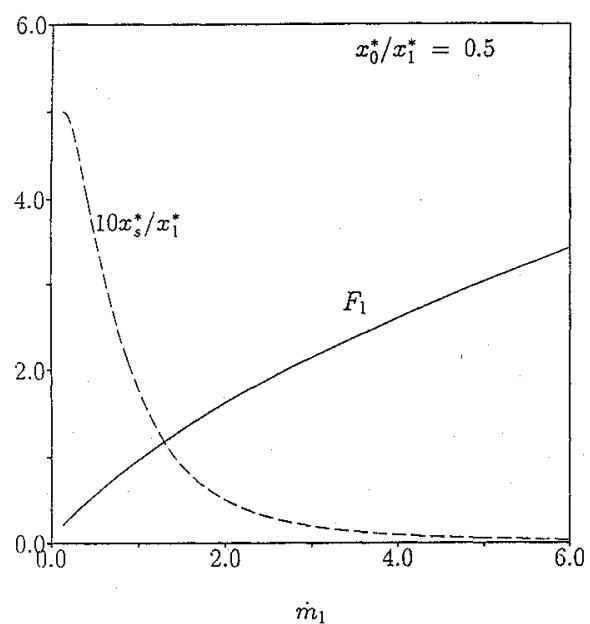

Fig. 6 Integrated scaled force change and nondimensional location of separation, as functions of scaled mass flow rate.

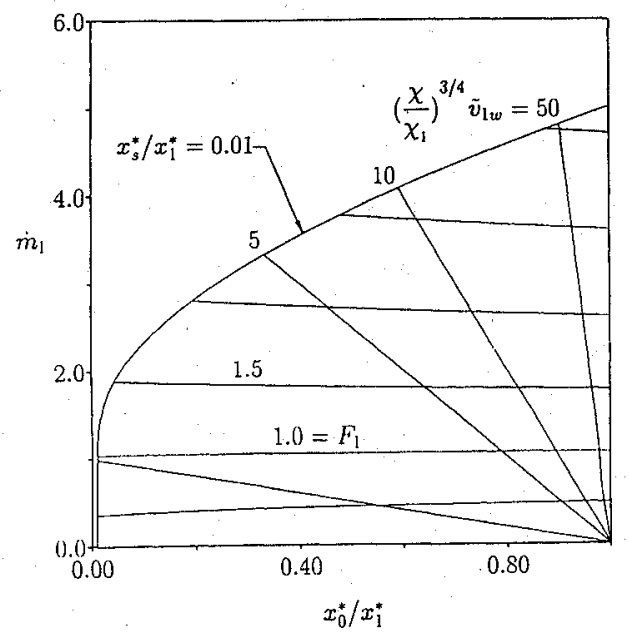

Fig. 7 Scaled rate of mass addition vs nondimensional location of slot leading edge, for constant values of scaled force change or scaled blowing velocity.

where the first term arises from the constant pressure in $x_{s}^{*}<x^{*}<$ $x_{0}^{*}$. From Eqs. (60) and (61), it is seen that $F_{1}$ is zero when $\tilde{v}_{1 w}=0$; $F_{1}$ then increases as $\tilde{v}_{1 w}$ increases, as in Fig. 6 . At the larger values of $\tilde{v}_{1 w}$ shown in the figure, the force change is approximately equal to the pressure increase in the plateau region multipled by the length of the separation region.

Figure 7 shows the required mass flow vs slot width for several values of integrated force. In the figure, the leading edge $x^{*}=x_{0}^{*}$ of the slot moves rearward from the wedge vertex as the coordinate $x_{0}^{*} / x_{1}^{*}$ increases from zero, and the slot width approaches zero as $x_{0}^{*} / x_{1}^{*}$ approaches one. The force change $F_{1}$ is seen to depend primarily on the mass flow $\dot{m}_{1}$, since the lines of constant force are nearly horizontal: if the slot width changes, but the mass flow is held constant, the variation in $F_{1}$ is very small. Lines of constant scaled blowing velocity $\left(\chi / \chi_{1}\right)^{\frac{3}{4}} \tilde{v}_{1 w}$ are also shown; these are straight lines, since the mass flow is proportional to the slot width. The boundary at the left of the figure is a curve for which the blowing velocity or slot width is large enough that the separation point $x^{*}=x_{s}^{*}$ is very close to the wedge vertex, at $x_{s}^{*}=0.01 x_{1}^{*}$.

Next it is supposed that gas is injected from two strips, defined by $x_{0}^{*}<x^{*}<x_{1}^{*}$ and $x_{2}^{*}<x^{*}<x_{3}^{*}$ (Fig. 8). For the first strip, the coordinates and scaled quantities are defined in the same way as in Eqs. (44-48), but with the superscript (1) added to the notation to distinguish quantities which refer to the first strip; e.g., $\tilde{x}^{(1)}$ and $\tilde{p}_{1}^{(1)}$ are written in place of $\tilde{x}$ and $\tilde{p}_{1}$, respectively. At the trailing edge of the first strip, the value $\tilde{x}_{f}^{(1)}=\left(x_{1}^{*}-x_{0}^{*}\right) /\left(x_{0}^{*}-x_{s}^{*}\right)$ of the streamwise coordinate $\tilde{x}^{(1)}$ (hence the location of the first separation point) is 


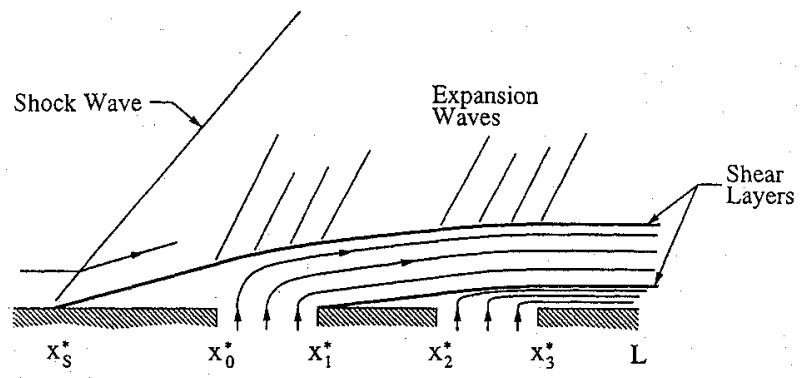

Fig. 8 Some flow details for blowing from two strips.

found from the condition

$$
\tilde{p}_{1}^{(1)}\left(\tilde{x}_{f}^{(1)}\right)=\tilde{p}_{1 f}^{(1)}, \quad 0<\tilde{p}_{1 f}^{(1)}<1
$$

where $\tilde{p}_{1 f}^{(1)}$ is to be determined.

If it is assumed that the flow from the first strip separates at $x_{1}^{*}$ and moves away from the surface with a constant slope, as depicted in Fig. 8, then the pressure is constant between $x_{1}^{*}$ and $x_{2}^{*}$; furthermore, the flow description for the second strip may be written in the same form as the first through an appropriate choice of scaled variables. The coordinates for the second strip are

$$
\begin{gathered}
\tilde{x}^{(2)}=\frac{x^{*}-x_{2}^{*}}{x_{2}^{*}-x_{1}^{*}} \\
\tilde{y}^{(2)}=\frac{y^{*}}{\tilde{\delta}_{0} \tilde{p}_{1 f}^{(1)}\left(x_{2}^{*}-x_{1}^{*}\right) /\left(x_{0}^{*}-x_{s}^{*}\right)}
\end{gathered}
$$

and expansions for the flow variables take the same form as in Eqs. (45-48), but with $\tilde{u}_{1}(\tilde{x}, \tilde{y}), \tilde{v}_{1}(\tilde{x}, \tilde{y}), \tilde{p}_{1}(\tilde{x}, \tilde{y})$, and $\tilde{T}_{w}$ replaced by $\left(\tilde{p}_{1 f}^{(1)}\right)^{\frac{1}{2}} \tilde{u}_{1}^{(2)}(\tilde{x}, \tilde{y}),\left(\tilde{p}_{1 f}^{(1)}\right)^{\frac{3}{2}} \tilde{v}_{1}^{(2)}(\tilde{x}, \tilde{y}), \tilde{p}_{1 f}^{(1)} \tilde{p}_{1}^{(2)}(\tilde{x}, \tilde{y})$, and $\tilde{T}_{w}^{(2)}$, respectively; with these substitutions, the governing equations may be written as in Eqs. (55) and (56). At the end of the second strip, it is assumed that the flow does not separate, so that

$$
\tilde{p}_{1}^{(2)}\left(\tilde{x}_{f}^{(2)}\right)=0
$$

where $\tilde{x}_{f}^{(2)}=\left(x_{3}^{*}-x_{2}^{*}\right) /\left(x_{2}^{*}-x_{1}^{*}\right)$.

If $\tilde{v}_{1 w}^{(1)}$ and $\tilde{v}_{1 w}^{(2)}$ are both constant, it follows from integration of Eq. (57) that the pressure distribution over each strip may be written in the implicit form

$$
\tilde{x}^{(i)}\left(\tilde{p}_{1}^{(i)} ; \tilde{v}_{1 w}^{(i)}\right)=\sum_{n=0}^{\infty} \frac{2}{(n+1)} A_{n}^{(i)}\left(1-\tilde{p}_{1}^{(i)}\right)^{(n+1) / 2}
$$

where the superscript $(i)$ denotes either strip one or two; $A_{0}^{(i)}=$ $2^{\frac{1}{2}} /\left(\pi \tilde{v}_{1 w}^{(i)}\right)$ and the coefficients $A_{n}^{(i)}$ satisfy the recursion relation (58). Evaluating this equation at the trailing edge of each strip gives

$$
\begin{gathered}
\left(x_{1}^{*}-x_{0}^{*}\right) /\left(x_{0}^{*}-x_{s}^{*}\right)=\tilde{x}^{(1)}\left(\tilde{p}_{1 f}^{(1)} ; \tilde{v}_{1 w}^{(1)}\right) \\
\left(x_{3}^{*}-x_{2}^{*}\right) /\left(x_{2}^{*}-x_{1}^{*}\right)=\tilde{x}^{(2)}\left(0 ; \tilde{v}_{1 w}^{(2)}\right)
\end{gathered}
$$

For a given slot geometry, Eq. (73) establishes the value of the scaled blowing velocity $\tilde{v}_{1 w}^{(2)}$. Given the ratio $v_{w}^{(2)} / v_{w}^{(1)}$ of blowing velocities, which may be expressed in terms of scaled quantities as

$$
\frac{v_{w}^{(2)}}{v_{w}^{(1)}}=\left(\frac{\tilde{T}_{w}^{(2)}}{\tilde{T}_{w}^{(1)}}\right)^{\frac{1}{2}}\left(\tilde{p}_{1 f}^{(1)}\right)^{\frac{3}{2}} \frac{\tilde{v}_{1 w}^{(2)}}{\tilde{v}_{1 w}^{(1)}}
$$

then a value of $\tilde{p}_{1 f}^{(1)}$ may be determined if the temperature ratio $\tilde{T}_{w}^{(2)} / \tilde{T}_{w}^{(1)}$ and the scaled blowing velocity $\tilde{v}_{1 w}^{(1)}$ are also specified. The location of the first separation point is then found from Eq. (72). Values of the parameters are to be selected so that $0<\tilde{p}_{1 f}^{(1)}<1$ and $\tilde{x}_{f}^{(1)}>0$.

Some pressure distributions for two strips are shown in Fig. 9. The lengths of the strips are taken to be equal, with the distance between the strips equal to twice the width of one strip. In terms of

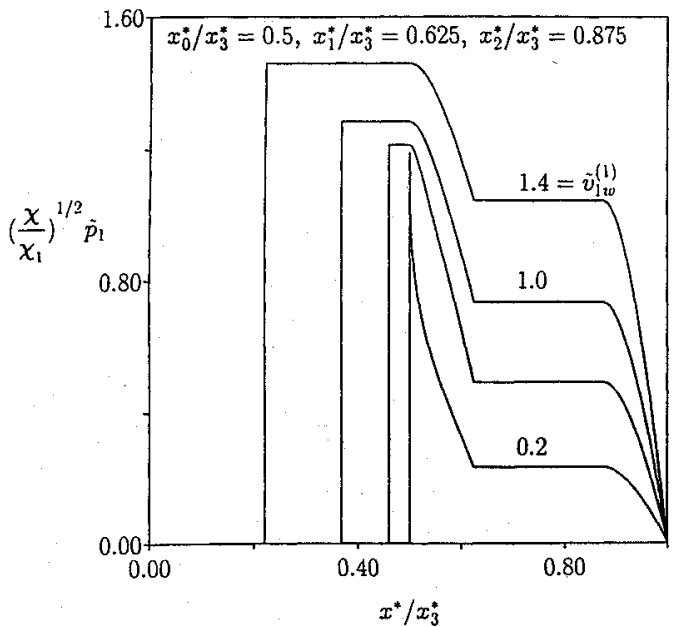

Fig. 9 Pressure distributions for blowing from two strips, for various rates of mass addition.

the distance $x_{3}^{*}$ from the wedge vertex to the end of the second strip, the first strip lies between $x^{*} / x_{3}^{*}=0.5$ and $x^{*} / x_{3}^{*}=0.625$, whereas the second strip extends from $x^{*} / x_{3}^{*}=0.875$ to $x^{*} / x_{3}^{*}=1.0$. The blowing velocities $v_{w}^{(1)}$ and $v_{w}^{(2)}$ are taken to be the same for the two strips as are the wall temperatures $\tilde{T}_{w}^{(1)}$ and $\tilde{T}_{w}^{(2)}$. As the mass flow is increased, the separation point is seen to move upstream, as expected, and the pressure rise at separation increases, since the Reynolds number $R e$ decreases and the interaction parameter $\chi$ increases. Thus, the solution is implicit in the sense that the scaled blowing velocity or mass flow is specified, and the corresponding dimensional values calculated after the location of separation has been determined.

\section{Concluding Remarks}

The solutions of Ref. 1 for strip blowing from a flat plate at supersonic speed have been extended relatively easily to wedge flow at high Mach number, if the hypersonic viscous interaction parameter is small. The flow near separation is described as a hypersonic free interaction for a cooled surface by extension and modification of results from Refs. 7-9; this gives the value for the pressure at the beginning of blowing. The dependence of the integrated force on the parameters is then determined for a particular parameter range.

If the surface were not cooled, the free-interaction solution for supersonic flow would suffice, provided that the viscous interaction for the undisturbed wedge flow is weak. For the cooled wall considered here, it is assumed that the wall temperature is small in comparison with the maximum temperature in the boundary layer. From Eq. (39) it then follows that the parameter $Q \chi^{\frac{1}{4}}$ must be sufficiently small. The present solution near separation would, however, have to be replaced by that of Ref. 10 if also $Q$ is small.

It has also been assumed that the flow does not separate at the end of the blowing region (or behind the second strip in the case of two strips). A proposal for describing the flow details near the end of blowing was given in Ref. 2, but the derivation seems based on an implicit assumption that separation does not occur. The present authors believe that a correct representation may include a shallow separation bubble, but thus far have not been able to complete a self-consistent flow description. Carter ${ }^{13}$ has attempted to simulate the flow near the strip trailing edge by computing solutions of the Navier-Stokes equations for a model problem with boundary conditions consistent with Ref. 2 ; in the cases considered, a slender separation bubble was observed. Although further work appears to be required for a complete description of the flow near the end of blowing, numerical solutions presented in Ref. 13 suggest that the choice $\tilde{p}_{1}\left(\tilde{x}_{1}\right)=0$ gives good accuracy for the surface pressure.

\section{Acknowledgment}

The authors would like to express their thanks to T. C. Adamson Jr. for many helpful discussions concerning this work. 


\section{References}

${ }^{1}$ Smith, F. T., and Stewartson, K., "Plate-Injection into a Separated Supersonic Boundary Layer," Journal of Fluid Mechanics, Vol. 58, Pt. 1, 1973, pp. 143-159.

${ }^{2}$ Stewartson, K., "Plate-Injection into a Separated Supersonic Boundary Layer. Part 2, The Transition Regions," Journal of Fluid Mechanics, Vol. 62 , Pt. 2, 1974, pp. 289-304.

${ }^{3}$ Cole, J. D., and Aroesty, J., "The Blowhard Problem-Inviscid Flows with Surface Injection," International Journal of Heat and Mass Transfer, Vol. 11, No. 7, 1968, pp. 1167-1183.

${ }^{4}$ Stewartson, K., and Williams, P. G., "Self-Induced Separation," Proceedings of the Royal Society of London, Series A, Vol. 312, 1969, pp. 181-206.

${ }^{5}$ Neiland, V. Y., "Theory of Laminar Boundary-Layer Separation in Supersonic Flow," Fluid Dynamics, Vol. 4, No. 4, 1969, pp. 33-35.

${ }^{6}$ Stewartson, K., and Williams, P. G., "Self-Induced Separation II," Mathematika, Vol. 20, Pt. 1, 1973, pp. 98-108.

${ }^{7}$ Brown, S. N., Stewartson, K., and Williams, P. G., "Hypersonic SelfInduced Separation," Physics of Fluids, Vol. 18, No. 6, 1975, pp. 633-639.
${ }^{8}$ Neiland, V. Y., "Boundary-Layer Separation on a Cooled Body and Interaction with a Hypersonic Flow," Fluid Dynamics, Vol. 8, No. 6, 1973, pp. 931-939.

${ }^{9}$ Brown, S. N., Cheng, H. K., and Lee, C. J., "Inviscid-Viscous Interaction on Triple-Deck Scales in a Hypersonic Flow with Strong Wall Cooling," Journal of Fluid Mechanics, Vol. 220, 1990, pp. 309-337.

${ }^{10}$ Kerimbekov, R. M., Ruban, A. I., and Walker, J. D. A., "Hypersonic Boundary-Layer Separation On a Cold Wall," Journal of Fluid Mechanics, Vol. 274, 1994, pp. 163-195.

${ }^{11}$ Seddougui, S. O., Bowles, R. I., and Smith, F. T., "Surface-Cooling Effects on Compressible Boundary-Layer Stability," Institute for Computer Applications in Science and Engineering, ICASE Rept. 90-19, NASA Langley Research Center, Hampton, VA, Feb. 1990.

${ }^{12}$ Stewartson, K., The Theory of Laminar Boundary Layers in Compressible Fluids, Oxford Univ. Press, Oxford, England, UK, 1964.

${ }^{13}$ Carter, N. D., "Computation of Hypersonic Flows with Strong Surface Blowing," Ph.D. Thesis, Dept. of Aerospace Engineering, Univ. of Michigan, Ann Arbor, MI, May 1991.

\title{
Introduction to Dynamics and Controlof FlexibLeStructures
}

\author{
JOHN L. JUNKINS AND YOUDAN KIM
}

This new textbook is the first to blend two traditional disciplines: Engineering Mechanics and Control Engineering. Beginning with theory, the authors proceed through computation, to laboratory experiment, and present actual case studies to illustrate practical aerospace applications. SDCMO: Structural Dynamics and Control MATLAB ${ }^{\circledR}$ Operators and a set of exercises at the end of each chapter complement this important new teaching tool. A 100-page solutions manual is available for the convenience of the instructor.

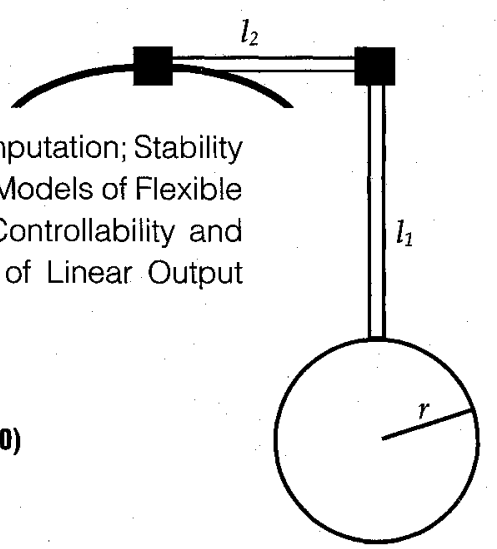

Place your order today! Call 1-800/682-AlAA

SAIA슬

American Institute of Aeronautics and Astronautics

Publications Customer Service, 9 Jay Gould Ct., P.O. Box 753, Waldorf, MD 20604 FAX 301/843-0159 Phone 1-800/682-2422 8 a.m. - 5 p.m. Eastern
Sales Tax: CA residents, $8.25 \% ; 0 \mathrm{D}, 6 \%$. For shipping and handling add $\$ 4.75$ for $1-4$ books (call) for rates for higher quantities). Orders under $\$ 100.00$ must be prepaid. Foreign orders must be prepaid and include a $\$ 20.00$ postal surcharge. Please allow 4 weeks for delivery. Prices are subject to change without notice. Returns will be accepted within 30 days. Non-U.S. residents are responsible for payment of any taxes required by their government. 